Zaid Saffo, DO, Weixia Guo, MD, MSc, Kylie Springer, MS, Kathleen Maksimowicz-McKinnon, DO, Vivek Kak, MD, John E. McKinnon, MD, MSc and Pallavi Bhargava*, MD

\title{
The role of tocilizumab therapy in critically ill patients with severe acute respiratory syndrome coronavirus 2
}

https://doi.org/10.1515/jom-2020-0292

Received November 10, 2020; accepted March 29, 2021;

published online July 12, 2021

\section{Abstract}

Context: Tocilizumab (TCZ), an interleukin-6 (IL-6) receptor antagonist, has been approved for use in rheumatoid arthritis and cytokine storm syndrome (CSS) associated with chimeric antigen receptor T cells treatment. Although TCZ is currently utilized in the treatment of critically ill coronavirus 2019 (COVID-19) patients, data on survival impact is minimal.

Objectives: To assess the mortality rate of patients presenting with COVID-19 who received TCZ for suspected CSS. Methods: This retrospective cohort study was conducted at Henry Ford Health System between March 10, 2020 and May 18, 2020. Data collection began in May 2020 and was completed in June 2020. Patients included in the study required hospital admission and had positive severe acute respiratory syndrome coronavirus 2 (SARS-CoV-2) polymerase chain reaction on nasopharyngeal swab. Eligibility criteria to receive TCZ, per hospital protocol, included any of the following: persistent fever, defined as $38.0^{\circ} \mathrm{C}$ for at least 6 hours; a diagnosis of the acute respiratory distress syndrome (ARDS); serum ferritin $\geq 1,000$ ( $\mathrm{ng} / \mathrm{mL}$ ) or doubling within 24 hours; D-Dimer $\geq 5$ (mg/L); serum lactate dehydrogenase $\geq 500$ (IU/L); or interlukin- 6 level $\geq 5$ times the

*Corresponding author: Pallavi Bhargava, MD, Division of Infectious Disease, Department of Internal Medicine, Henry Ford Health System, 2799 West Grand Blvd, Detroit, MI 48202, USA,

E-mail: pbharga1@hfhs.org

Zaid Saffo, DO, Weixia Guo, MD, MSc and Kathleen MaksimowiczMcKinnon, DO, Division of Rheumatology, Department of Internal Medicine, Henry Ford Health System, Detroit, Michigan, USA

Kylie Springer, MS, Department of Public Health Sciences, Henry Ford Health System, Detroit, Michigan, USA

Vivek Kak, MD and John E. McKinnon, MD, MSc, Division of Infectious Disease, Department of Internal Medicine, Henry Ford Health System, Detroit, Michigan, USA upper limit of normal. Dosing was initially determined by weight, then changed to a fixed $400 \mathrm{mg}$ per hospital protocol. A comparator cohort was created from patients with COVID-19 and ARDS who did not receive TCZ. Patient survival was analyzed using the Kaplan-Meier method and compared by log rank test. A multivariable cox regression was applied to evaluate the association between TCZ and mortality.

Results: One hundred and thirty patients were evaluated in the study, 54 (41.5\%) of whom received TCZ. Patients who received TCZ were younger (mean age, 63.8 vs. 69.4 years; $\mathrm{p}=0.0083$ ) and had higher body mass indices (mean, 33.9 vs. $30.4 ; \mathrm{p}=0.005$ ). Of the comorbid conditions evaluated, heart disease was more common in the comparator group than the TCZ group (27 patients [35.5\%] vs. 10 patients [18.5\%]; $p=0.034$ ). A Kaplan-Meier survival curve demonstrated no difference in survival between TCZ and comparator patients $(\log$ rank $\mathrm{p}=0.495)$. In the multivariable Cox regression model for mortality at 30 days, treatment with TCZ was not associated with decreased mortality (hazard ratio, 1.1; 95\% confidence interval, 0.53-2.3; $\mathrm{p}=0.77$ ). Lower mean C-reactive protein (CRP) levels were demonstrated within 48 hours of disposition in the TCZ group (mean TCZ, 4.9 vs. mean comparator, $13.0 ; p=<0.0001$ ).

Conclusions: In this cohort study, no difference in survival was observed in critically ill patients treated with TCZ.

Keywords: coronavirus; COVID-19; mortality; survival; tocilizumab.

The coronavirus 2019 (COVID-19) pandemic caused by severe acute respiratory syndrome coronavirus 2 (SARS-CoV-2) has ravaged the world since it was initially reported in China in December of 2019 [1]. As of April 2021, over 157 million people have been infected and over three million deaths have been reported worldwide [2]. Based on published data, it is estimated that $81 \%$ of symptomatic people develop mild to moderate disease, $14 \%$ develop severe disease, and 5\% develop critical disease associated with respiratory failure, 
shock, or multiorgan system failure [3]. The mortality rate in patients admitted to the intensive care unit (ICU) has been estimated between 21 and 48.7\% [4-6].

The reasons certain patients with COVID-19 progress to severe disease remains unclear. Some research $[7,8]$ has suggested that the progression of the disease to severe form could be caused by cytokine storm syndrome (CSS). CSS is clinically characterized by cytopenia, hyperferritinemia, fever, acute respiratory distress syndrome (ARDS), and if untreated, death $[7,9,10]$. Studies of the previous coronavirus strains, SARS-CoV and Middle East Respiratory Syndrome coronavirus (MERS-CoV), also demonstrated the increased production and release of proinflammatory cytokines including interleukin 6 (IL-6), interleukin $1 \beta$ (IL-I $\beta$ ), interferon $\beta$ (IFN- $\beta$ ), interleukin 12 (IL-12), and tumor necrosis alpha (TNF- $\alpha)[11,12]$.

The use of corticosteroids and other immunosuppressive therapies in the treatment of infectious conditions associated with CSS or similar proinflammatory syndromes remains limited to patients with bacterial [13] and tuberculous meningitis [14], Pneumocystis jiroveci pneumonia (PJP) [15], and immune reconstitution inflammatory syndrome (IRIS) [16] with HIV. Its use is a controversial and unsettled topic in other conditions. A systematic review [17] of 29 SARS-CoV studies evaluating corticosteroid use determined that 25 were inconclusive and four demonstrated potential harm. A retrospective analysis [18] of 213 patients admitted to our institution with COVID-19 demonstrated that an early course of methylprednisolone initiated at a median time of 2 days after presentation reduced rates of death, ICU transfer, and mechanical ventilation. In a randomized controlled trial [19] of 6,425 patients with COVID-19, those requiring oxygen support who received dexamethasone had a lower 28 day mortality rate than those who received usual ( 22.9 vs. $25.7 \%$ ).

As the pandemic has progressed with only corticosteroids $[17,18]$ as consistently efficacious treatment against COVID-19 in certain patients, the search for other interventions to benefit the most severely affected and at risk patients has broadened. IL- 6 blockade has been proposed as a potential therapeutic option in patients with COVID-19 in the setting of CSS [8]. Tocilizumab (TCZ) is an IL-6 receptor antagonist used in the treatment of rheumatoid arthritis and giant cell arteritis and has also been approved for treatment of CSS associated with chimeric antigen receptor $\mathrm{T}$ cells [20]. Several small, nonrandomized studies [8, 21-26] evaluating the use of TCZ for COVID-19 have demonstrated varying results. Recent data from five randomized controlled trials [27-31] also demonstrated varying results.

TCZ was added to the institutional treatment guidelines at Henry Ford Health System for patients hospitalized with SARS-CoV-2 infection in March 2020. The purpose of this study was to investigate the impact of TCZ therapy on mortality in critically ill patients at suburban and semirural community based centers.

\section{Methods}

\section{Study population}

The study was approved by the Institutional Review Board of Henry Ford Health System (\#13860). A waiver for consent and approval to include vulnerable population data (prisoners) was obtained.

This retrospective cohort study included adults (18 years of age or older) who were hospitalized at Henry Ford West Bloomfield (West Bloomfield, Michigan) and Henry Ford Allegiance Hospital (Jackson, Michigan) between March 10, 2020 and May 18, 2020 with COVID-19. Data collection started in May 2020 and was completed in June 2020. All patients had positive test results for SARS-CoV-2 infection confirmed via polymerase chain reaction on nasopharyngeal swab. Patients were included if they had received TCZ for suspected CSS associated with COVID-19 during the study period. TCZ use for COVID-19 was off label and the eligibility criteria to receive TCZ, per hospital protocol, included any of the following: persistent fever, defined as $38.0{ }^{\circ} \mathrm{C}$ for at least 6 hours; a diagnosis of ARDS; serum ferritin $\geq 1,000(\mathrm{ng} / \mathrm{mL})$ or doubling within 24 hours; D-dimer $\geq 5$ (mg/ L); serum lactate dehydrogenase (LDH) $\geq 500$ (IU/L); or interlukin-6 level $\geq 5$ times the upper limit of normal. Patients were not candidates for TCZ if they had any of the following: aspartate aminotransferase/ alanine aminotransferase (ALT) $\geq 5$ times the upper limit of normal; neutrophils $<0.5(\mathrm{~K} / \mu \mathrm{L})$; platelets $<50(\mathrm{~K} / \mu \mathrm{L})$; or clinical, laboratory, or microbiologic evidence of concurrent bacterial or fungal infection. The decision to administer TCZ was made by an infectious disease specialist (authors P.B., V.K., and other non authors), based on the eligibility criteria described. TCZ was initially given as dose of $8 \mathrm{mg}$ per $\mathrm{kg}$ of patient weight; however, due to drug shortage related to nationally increased utilization of TCZ for severe COVID-19, our hospital protocol was amended on March 30, 2020 to a fixed $400 \mathrm{mg}$ single intravenous dose. Patients were eligible to receive a second dose if they had persistent fever.

Patients with COVID-19 and ARDS who did not receive TCZ were placed in a comparator group. Patients in both groups were excluded from the study if they were transferred to different institutions from the emergency department, died within 48 hours of admission, or were enrolled in hospice care.

Again, protocols for corticosteroid treatment for COVID-19 were incorporated into the Henry Ford Health System guidelines on March 20, 2020. Methylprednisolone was recommended for hypoxic patients who required supplemental oxygen; the recommended treatment regimen was 3-7 days with weight dependent dosing (40 mg twice daily for $100 \mathrm{~kg}$ or less; $60 \mathrm{mg}$ twice daily for $101 \mathrm{~kg}-160 \mathrm{~kg} ; 80 \mathrm{mg}$ twice daily for $>160 \mathrm{~kg}$ ). The decision to use corticosteroid (and duration for which it was given) was made by each patient's primary medical team.

\section{Outcomes}

The primary outcome of this study was mortality at 30 days. Secondary outcomes were hospital length of stay, ICU length of stay, duration on 
mechanical ventilation, changes in serologic markers of inflammation (ferritin, C-reactive protein [CRP], LDH, D-dimer, total white blood cell [WBC] count, platelet count), and serologic indicators of organ dysfunction (serum creatinine, liver function studies, creatine phosphokinase, and troponin) associated with CSS.

\section{Data collection}

Data were collected manually from the electronic medical record and entered into a standardized Excel form (Microsoft, Inc.). A quality control protocol was developed by the research team prior to data collection to ensure a uniform abstraction and entry process. Data collected included demographics (sex, age, body mass index [BMI], comorbidities [hypertension, diabetes, chronic obstructive pulmonary disease, asthma, renal disease, heart disease, immunocompromised status, smoking], and clinical parameters [highest respiratory support]). The presence of renal disease was categorized as chronic kidney disease stage III-IV or dialysis dependent. An immunocompromised state was defined as infection with human immunodeficiency virus or current immunosuppressive therapy. The initial Sequential Organ Failure Assessment (SOFA) and Acute Physiology and Chronic Health Evaluation (APACHE) II scores for patients admitted to the ICU was manually calculated using data from the ICU admission date. APACHE II scores were also calculated at the time of first and second TCZ administration for the group of patients who received two doses.

Laboratory data collected for this study included ferritin, CRP, creatinine, creatinine phosphokinase (CPK), WBC count, platelets, aspartate transaminase (AST), ALT, absolute neutrophil to lymphocyte ratio (NLR), D-dimer, high sensitivity troponin, procalcitonin, and a $\mathrm{PaO} 2 / \mathrm{FiO} 2(\mathrm{P} / \mathrm{F})$ ratio. The earliest laboratory tests during the first 24 hours after admission were recorded for this study, as were the latest values within 48 hours of discharge. Ferritin and D-dimer values were categorized into ranges as the two hospital laboratories used different references for normal values. All laboratory tests were performed at the discretion of the treating physician.

Treatment data included corticosteroid use (prednisone, methylprednisolone, and dexamethasone) with total duration in days; use of agents potentially targeting SARS-CoV-2 (hydroxychloroquine alone or in combination with azithromycin); use of other antimicrobials, convalescent plasma, intravenous immunoglobulin (IVIG); and anticoagulation use (prophylaxis or therapeutic). Patients in the TCZ group were designated as having antithrombotic therapy if they received at least 3 days of full anticoagulation.

Other clinical events recorded for all patients included use of vasopressors, acute kidney injury, need for continuous renal replacement therapy (CRRT) or hemodialysis, need for oxygen at discharge, cardiac arrest, and presence of positive blood and sputum/tracheal aspirate. CRRT or hemodialysis were initiated at the discretion of the treating nephrologist. In the TCZ group, only infections that occurred after TCZ administration were recorded. Instances of infection documented by an infectious disease specialist were used to distinguish true infection from colonization. Thirty days of follow up in the TCZ group was recorded from the time of TCZ administration.

\section{Statistical analysis}

All continuous data are reported using mean and standard deviation, count, median, and range, while categorical data are reported as counts and column percentages (n [\%]). Univariate two group comparisons were performed using t-tests for normally distributed continuous variables, Wilcoxon rank sum for continuous variables that were skewed, and chi-square or Fisher's exact test for categorical variables. Statistical significance was set at $\mathrm{p}<0.05$.

Patient mortality was analyzed using the Kaplan-Meier method and compared by log rank test. A multivariate cox regression was applied to evaluate the association between TCZ and mortality. Variables with a univariate $\mathrm{p}<0.10$ were candidates for inclusion in the final model. Variables included in the analysis were demographics (e.g., age, sex), treatment modalities (e.g., antimicrobial, anticoagulation), laboratory data (CRP, NLR, D-dimer, and WBC), and duration of corticosteroid therapy. TCZ use was accounted for as a time varying exposure to mitigate immortal time bias. The final model was obtained via forward selection process with significance level for entry set at $\mathrm{p}<0.05$. All analyses were performed using SAS 9.4 (SAS Institute Inc.) and SPSS 27 (IBM Corp.).

\section{Results}

During the study period, 151 patients were hospitalized with COVID-19 ARDS at the participating hospitals and these consecutive patients were evaluated for inclusion. Of those, 54 (35.7\%) received TCZ during the study period and all 54 (100.0\%) were included. Ninety seven comparator patients $(64.2 \%)$ were identified and seventy six (50.3\%) were included.

\section{Demographic characteristics}

Data for patient demographics, comorbid conditions, and severity of illness are presented in Table 1 . The mean age (standard deviation, SD) was 63.8 years (SD, \pm 11.6 ; range, 29-87 years) in the TCZ group and 69.4 years (SD, \pm 11.5 ; range, $43-90$ years) in the comparator group ( $\mathrm{p}=<0.01$ ). In the TCZ group, 39 patients $(72.2 \%)$ were male and 24 (28.9\%) were female; in the comparator group, 54 patients $(71.1 \%)$ were male and 24 (28.9\%) were female. Of the comorbid conditions evaluated in this study, heart disease was more common in the comparator group than the TCZ group (27 patients [35.5\%] vs. 10 patients [18.5\%]; $\mathrm{p}=0.03)$. The majority of patients required invasive ventilation during their hospitalization (TCZ group, 47 $[87.0 \%]$ vs. comparator group, 61 [80.3\%] patients; $\mathrm{p}=0.43$ ). The mean APACHE II score in TCZ survivors (22; $40.7 \%)$ was $17.5(\mathrm{SD}, \pm 5.9)$ compared with $21(\mathrm{SD}, \pm 8)$ in patients who received TCZ but did not survive $(21 ; 38.9 \%)$ $(\mathrm{p}=0.13)$. In patients who received a second dose of TCZ (17; 31.5\%), the mean APACHE II score at first dose was $23.2(\mathrm{SD}, \pm 8.40)$ and $25.0(\mathrm{SD}, \pm 8.71)$ at second dose $(p=0.11)$. All patients $(54 ; 100.0 \%)$ in the TCZ and 
comparator groups received corticosteroids (primarily intravenous methylprednisolone; data not available). There was no statistically significant difference between the TCZ group and the comparator group in antibiotic usage (TCZ group, 47 patients [87.0\%] vs. comparator group, 66 patients [86.8\%]; $\mathrm{p}=0.97$ ), convalescent plasma (TCZ group, four patients [14.8\%] vs. comparator group, nine patients [11.8\%]; $\mathrm{p}=0.62$ ), or antimicrobial use [hydroxychloroquine (TCZ group, 30 patients [55.5\%] vs. comparator group, 46 patients [60.5\%]), $\mathrm{p}=0.84$; hydroxychloroquine with azithromycin (TCZ group, 13 patients [24.1\%] vs. comparator group, 17 patients [22.4\%], $\mathrm{p}=0.84$ ), none (TCZ group, 11 patients [20.4\%] vs. comparator group, 13 patients [17.1\%], $\mathrm{p}=0.84)]$. The difference in rate of anticoagulation use between groups was not statistically significant with either prophylaxis dose (TCZ group, 20 patients [37.0\%] vs. comparator group, 24 patients [31.6\%]; $\mathrm{p}=0.31$ ) or therapeutic dose (TCZ group, 34 patients [63.0\%] vs. comparator group, 50 patients [65.8\%]; $\mathrm{p}=0.31)$. Four patients $(7.4 \%)$ in the TCZ group received IVIG. The majority of patients $(38 ; 70.4 \%)$ received a $400 \mathrm{mg}$ TCZ dose, while two (3.7\%) received a $600 \mathrm{mg}$ TCZ dose and 14 (25.9\%) received an $800 \mathrm{mg}$ dose. For patients who did not receive TCZ, bacterial infection was documented as a reason in $14(18.4 \%)$, while other documented reasons not related to infection were given for $16(21.1 \%)$ and no documented reason was given for 46 $(60.5 \%)$.

\section{Laboratory data}

Laboratory results data are presented in Table 2. Differences in admission data did not rise to the level of statistical significance between groups for any category except LDH (TCZ group, 462.6 [SD, \pm 209.2$]$ vs. comparator group, 394.0 [SD, \pm 186.5 ]; $p=0.03$ ). Within 48 hours of discharge, mean CRP level was lower in the TCZ group than the comparator group (TCZ, $4.9 \pm 6.0$ vs. comparator, $13.0 \pm 12.0 ; p=<0.01$ ). Mean LDH values within 48 hours of discharge remained higher in the TCZ group than in the comparator group (TCZ, 525.2 [SD, \pm 440.4$]$ vs. comparator, $444.1[\mathrm{SD}, \pm 494.4] ; \mathrm{p}=0.02$ ). There was no difference in the final ratio of arterial oxygen concentration to the fraction of inspired oxygen $(\mathrm{P} / \mathrm{F})$ between the two groups $(\mathrm{p}=0.12)$. The mean IL-6 level was higher among smokers (58; 52.3\%) than nonsmokers $(53 ; 47.7 \%)$, though the difference was not statistically significant (smokers, 89.88 [SD, \pm 163.7$]$ vs. nonsmokers, 71.85 [SD, \pm 123.2 ]; $\mathrm{p}=0.45$ ).
Table 1: Demographics and comorbid conditions between tocilizumab (TCZ) and comparator groups.

\begin{tabular}{|c|c|c|c|}
\hline Characteristics & $\begin{array}{r}\text { TCZ group } \\
(\mathrm{N}=54)\end{array}$ & $\begin{array}{r}\text { Comparator } \\
\text { group } \\
(\mathrm{N}=76)\end{array}$ & $\begin{array}{l}\text { p- } \\
\text { Value }\end{array}$ \\
\hline Age, years, mean \pm SD & $63.8 \pm 11.6$ & $69.4 \pm 11.5$ & $<0.01$ \\
\hline $\mathrm{BMI}, \mathrm{kg} / \mathrm{m}^{2}$, mean $\pm \mathrm{SD}$ & $33.9 \pm 7.9$ & $30.4 \pm 6.2$ & $<0.01$ \\
\hline \multicolumn{4}{|l|}{ Sex } \\
\hline Female & 15 (27.8\%) & 22 (28.9\%) & 0.88 \\
\hline Male & 39 (72.2\%) & $54(71.1 \%)$ & 0.88 \\
\hline \multicolumn{4}{|l|}{ Ethnicity, n (\%) } \\
\hline White & $24(44.4 \%)$ & $47(61.8 \%)$ & 0.07 \\
\hline Black & $25(46.3 \%)$ & $28(36.8 \%)$ & \\
\hline Asian & $2(3.7 \%)$ & $0(0.0 \%)$ & \\
\hline Unknown & $3(5.6 \%)$ & $1(1.3 \%)$ & \\
\hline $\begin{array}{l}\text { Interleukin } 6 \text { level, } \mathrm{pg} / \mathrm{mL} \text {, } \\
\text { mean } \pm \mathrm{SD}\end{array}$ & $108.8 \pm 179$ & $62.3 \pm 105.3$ & 0.07 \\
\hline SOFA score, mean \pm SD & $5.7 \pm 2.2$ & $6.0 \pm 3.2$ & 0.96 \\
\hline $\begin{array}{l}\text { Symptom onset to admission, } \\
\text { days, mean } \pm \text { SD }\end{array}$ & $6.9 \pm 3.4$ & $7.1 \pm 4.4$ & 0.91 \\
\hline $\begin{array}{l}\text { Symptom onset to TCZ dose, } \\
\text { days, mean } \pm \text { SD }\end{array}$ & $12.6 \pm 4.69$ & & \\
\hline $\begin{array}{l}\text { Admission to TCZ, days, } \\
\text { mean } \pm \text { SD }\end{array}$ & $5.9 \pm 3.77$ & & \\
\hline $\begin{array}{l}\text { Corticosteroid duration, days, } \\
\text { mean } \pm \text { SD }\end{array}$ & $6.5 \pm 3.1$ & $6.4 \pm 2.7$ & 0.98 \\
\hline $\begin{array}{l}\text { Admission to corticosteroid } \\
\text { initiation, mean } \pm \text { SD d } \\
\text { Highest oxygen need, } n(\%)\end{array}$ & $0.72 \pm 1.38$ & $0.77 \pm 1.7$ & 0.85 \\
\hline Invasive vent & 47 (87.0\%) & $61(80.3 \%)$ & 0.43 \\
\hline Noninvasive vent & $3(5.6 \%)$ & $3(3.9 \%)$ & \\
\hline High flow & $3(5.6 \%)$ & $11(14.5 \%)$ & \\
\hline Nasal cannula & $1(1.9 \%)$ & $1(1.3 \%)$ & \\
\hline Comorbid conditions, n (\%) & $\begin{array}{r}\text { TCZ group } \\
(\mathrm{N}=54)\end{array}$ & $\begin{array}{r}\text { Comparator } \\
\text { group } \\
(\mathrm{N}=76)\end{array}$ & $\begin{array}{l}\mathrm{p}- \\
\text { Value }\end{array}$ \\
\hline Hypertension & $43(79.6 \%)$ & $55(72.4 \%)$ & 0.34 \\
\hline Diabetes & $26(48.1 \%)$ & 27 (35.5\%) & 0.15 \\
\hline Asthma & 7 (13.0\%) & $14(18.4 \%)$ & 0.41 \\
\hline $\begin{array}{l}\text { Chronic obstructive pulmonary } \\
\text { disease }\end{array}$ & $9(16.7 \%)$ & $18(23.7 \%)$ & 0.33 \\
\hline Cancer & $6(11.1 \%)$ & $18(23.7 \%)$ & 0.07 \\
\hline \multicolumn{4}{|l|}{ Renal disease } \\
\hline CKD III-V & $9(16.7 \%)$ & $18(23.7 \%)$ & 0.49 \\
\hline ESRD & 1 (1.9\%) & $3(3.9 \%)$ & \\
\hline $\begin{array}{l}\text { Heart failure or coronary artery } \\
\text { disease }\end{array}$ & $10(18.5 \%)$ & 27 (35.5\%) & 0.03 \\
\hline Immunocompromised status & $5(9.3 \%)$ & $5(6.6 \%)$ & 0.57 \\
\hline \multicolumn{4}{|l|}{ Smoking status } \\
\hline Prior user & $23(45.1 \%)$ & $39(52.7 \%)$ & 0.09 \\
\hline Active user & $3(5.9 \%)$ & 0 (0.0\%) & \\
\hline Never smoker & $25(49.0 \%)$ & $35(47.3 \%)$ & \\
\hline
\end{tabular}

BMI, body mass index; CKD, chronic kidney disease; ESRD, end stage renal disease; SD, standard deviation; SOFA, Sequential Organ Failure Assessment; TCZ, tocilizumab. 
Table 2: Laboratory data.

\begin{tabular}{|c|c|c|c|}
\hline Laboratory value & Tocilizumab group $(\mathrm{N}=54)$ & Comparator group $(\mathrm{N}=76)$ & p-Value \\
\hline \multicolumn{4}{|c|}{$\mathrm{CRP}, \mathrm{mg} / \mathrm{dL}$, mean $\pm \mathrm{SD}$} \\
\hline Initial & $13.2 \pm 7.4$ & $12.6 \pm 6.4$ & 0.65 \\
\hline Final & $4.9 \pm 6.0$ & $13.0 \pm 12.0$ & $<0.01$ \\
\hline \multicolumn{4}{|c|}{$\mathrm{N} / \mathrm{L}$ ratio, mean $\pm \mathrm{SD}$} \\
\hline Initial & $8.6 \pm 6.4$ & $10.2 \pm 8.2$ & 0.32 \\
\hline Final & $12.5 \pm 14.3$ & $16.5 \pm 18.7$ & 0.21 \\
\hline \multicolumn{4}{|c|}{ WBC, K/uL, mean \pm SD } \\
\hline Initial & $7.7 \pm 3.4$ & $8.3 \pm 5.5$ & 0.75 \\
\hline Final & $15.3 \pm 11.6$ & $12.5 \pm 7.1$ & 0.56 \\
\hline \multicolumn{4}{|c|}{ Creatinine, $\mathrm{mg} / \mathrm{dL}$, mean $\pm \mathrm{SD}$} \\
\hline Initial & $1.8 \pm 2.1$ & $1.7 \pm 1.5$ & 0.88 \\
\hline Final & $2.0 \pm 2.1$ & $1.7 \pm 1.5$ & 0.98 \\
\hline \multicolumn{4}{|c|}{$\mathrm{CPK}, \mathrm{IU} / \mathrm{L}$, mean $\pm \mathrm{SD}$} \\
\hline Initial & $535.3 \pm 678.5$ & $705.8 \pm 1,162.9$ & 0.18 \\
\hline Final & $403.1 \pm 750.4$ & $245.2 \pm 545.8$ & 0.09 \\
\hline \multicolumn{4}{|c|}{$\mathrm{LDH}, \mathrm{IU} / \mathrm{L}$, mean $\pm \mathrm{SD}$} \\
\hline Initial & $462.6 \pm 209.2$ & $394.0 \pm 186.5$ & 0.03 \\
\hline Final & $525.2 \pm 440.4$ & $444.1 \pm 494.4$ & 0.02 \\
\hline \multicolumn{4}{|c|}{$\mathrm{ALT}, \mathrm{IU} / \mathrm{L}$, mean $\pm \mathrm{SD}$} \\
\hline Initial & $50.3 \pm 67.6$ & $32.6 \pm 22.2$ & 0.15 \\
\hline Final & $145.6 \pm 491.7$ & $54.88 \pm 55.8$ & 0.11 \\
\hline \multicolumn{4}{|c|}{ AST, IU/L, mean $\pm S D$} \\
\hline Initial & $71.7 \pm 81.3$ & $53.5 \pm 37.6$ & 0.18 \\
\hline Final & $225.6 \pm 1,062.5$ & $85.25 \pm 274.6$ & 0.06 \\
\hline \multicolumn{4}{|c|}{ Procalcitonin, $\mathrm{ng} / \mathrm{mL}$, mean $\pm \mathrm{SD}$} \\
\hline Initial & $0.5 \pm 0.6$ & $1.5 \pm 6.0$ & 0.88 \\
\hline Final & $1.5 \pm 3.3$ & $4.0 \pm 13.7$ & 0.89 \\
\hline \multicolumn{4}{|c|}{ Troponin, $\mathrm{ng} / \mathrm{L}$, mean $\pm \mathrm{SD}$} \\
\hline Initial & $49.9 \pm 153.5$ & $58.9 \pm 147.4$ & 0.88 \\
\hline Final & $86.4 \pm 289.6$ & $177.3 \pm 700.8$ & 0.51 \\
\hline \multicolumn{4}{|c|}{$\mathrm{P} / \mathrm{F}$ ratio, mean $\pm \mathrm{SD}$} \\
\hline Initial & $141.08 \pm 94.8$ & $151.31 \pm 95.7$ & 0.18 \\
\hline Nadir & $78.07 \pm 30.7$ & $97.51 \pm 38.2$ & $<0.01$ \\
\hline Peak & $244.82 \pm 93.9$ & $284.61 \pm 128.9$ & 0.11 \\
\hline Final & $136.28 \pm 62.0$ & $166.28 \pm 88.8$ & 0.12 \\
\hline \multicolumn{4}{|c|}{ D-dimer, peak (ug/mL), n (\%) } \\
\hline $0-0.8$ & $4(7.5 \%)$ & $2(2.7 \%)$ & 0.11 \\
\hline $0.81-3.10$ & $14(26.4 \%)$ & $26(35.1 \%)$ & \\
\hline $3.11-9.99$ & 8 (15.1\%) & $20(27.0 \%)$ & \\
\hline$>10$ & $27(50.9 \%)$ & $26(35.1 \%)$ & \\
\hline \multicolumn{4}{|c|}{ Ferritin, initial (ng/mL), n (\%) } \\
\hline$<336$ & $6(12.2 \%)$ & $20(29.9 \%)$ & 0.14 \\
\hline $336-999$ & 19 (38.8\%) & $24(35.8 \%)$ & \\
\hline 1,000-1999 & $13(26.5 \%)$ & $12(17.9 \%)$ & \\
\hline$>2000$ & $11(22.4 \%)$ & $11(16.4 \%)$ & \\
\hline \multicolumn{4}{|c|}{ Ferritin, final (ng/mL), n (\%) } \\
\hline$<336$ & $5(11.1 \%)$ & $14(23.3 \%)$ & 0.19 \\
\hline $336-999$ & $21(46.7 \%)$ & $22(36.7 \%)$ & \\
\hline 1,000-1999 & 7 (15.6\%) & $14(23.3 \%)$ & \\
\hline$>2000$ & $12(26.7 \%)$ & $10(16.7 \%)$ & \\
\hline
\end{tabular}

ALT, alanine aminotransferase; AST, aspartate aminotransferase; CPK, creatine phosphokinase; CRP, C-reactive protein; LDH, lactic acid dehydrogenase; N/R ratio, absolute neutrophil to lymphocyte ratio; $\mathrm{P} / \mathrm{F}$ ratio, ratio of arterial oxygen concentration to the fraction of inspired oxygen; SD, standard deviation; WBC, white blood cell. 


\section{Outcomes}

TCZ did not significantly improve overall survival or other secondary outcome measures (Figure 1; Table 3). At 30 days, 22 patients (40.1\%) in the TCZ group had survived compared with $33(43.4 \%)$ in the comparator group $(\mathrm{p}=0.76)$. There was a trend toward longer mean length of stay in the TCZ group, but the difference was not statistically significant (TCZ, 18.4 [SD, \pm 11.2$]$ vs. comparator, 15.4 [SD, \pm 7.9 ] days; $\mathrm{p}=0.09$ ). Survival analysis revealed no statistically significant difference in mean hospital length of stay (TCZ group, $21.8[\mathrm{SD}, \pm 14.6]$ vs. comparator group, 18.4 [SD, \pm 8.1$]$ days; $\mathrm{p}=0.29$ ) or mean duration on ventilator (TCZ group, 10.6 $[S D, \pm 6.5]$ vs. comparator group, $7.8[S D, \pm 5.1]$ days; $\mathrm{p}=0.15$ ). Thirteen patients $(24.1 \%)$ in the TCZ group received renal replacement therapy compared with two $(2.6 \%)$ in the comparator group $(\mathrm{p}<0.01)$. The difference in the number of patients who had positive blood culture (TCZ group, four patients [7.4\%] vs. comparator group, seven patients [9.2\%]; $p=0.41$ ) was not statistically significant between groups. The most common blood stream pathogen in the TCZ group was candida albicans (three patients; $75.0 \%$ of positive cultures), whereas gram positive isolates were the most common in the comparator arm (six patients; $75.0 \%$ of positive cultures). Pseudomonas aeruginosa (10 patients [22.0\% of 45 total respiratory isolates]) and Staphylococcus aureus (10 patients [22.0\%] of 45 total respiratory isolates) were the most common causes of secondary pneumonia. Detailed superinfection data stratified by group are presented in the Supplementary Material.

In the multivariate Cox regression model for mortality at 30 days for the entire sample, treatment with TCZ was not associated with decreased mortality (hazard ratio
Table 3: Clinical events.

\begin{tabular}{lrrr}
\hline Characteristics, $\mathbf{n}$ (\%) & $\begin{array}{r}\text { Tocilizumab } \\
(\mathbf{N}=54)\end{array}$ & $\begin{array}{r}\text { Comparator } \\
(\mathbf{N}=76)\end{array}$ & $\begin{array}{r}\mathbf{p}- \\
\text { Value }\end{array}$ \\
\hline Survival at 30 days & $22(40.1 \%)$ & $33(43.4 \%)$ & 0.76 \\
Survival at 14 days & $31(57.4 \%)$ & $44(57.9 \%)$ & 0.95 \\
Vasopressor use & $35(64.8 \%)$ & $44(57.9 \%)$ & 0.43 \\
Acute kidney injury & $35(64.8 \%)$ & $46(60.5 \%)$ & 0.62 \\
Hemodialysis/CRRT & $13(24.1 \%)$ & $2(2.6 \%)$ & $<0.01$ \\
Cardiac arrest & $4(7.6 \%)$ & $3(3.9 \%)$ & 0.46 \\
CVA & $2(3.8 \%)$ & $1(1.3 \%)$ & 0.57 \\
Bleeding & $13(24.1 \%)$ & $11(14.5 \%)$ & 0.16 \\
Discharged with oxygen & $10(50.0 \%)$ & $12(38.7 \%)$ & 0.43 \\
Infection & & & \\
$\quad$ Blood stream infection & $4(7.4 \%)$ & $7(9.2 \%)$ & 0.41 \\
$\quad$ Pulmonary infection (endotra- & $14(25.9 \%)$ & $23(30.3 \%)$ & 0.71 \\
$\quad$ cheal aspirates/sputum) & & & \\
\hline Length of stay, days, mean \pm SD & & & \\
\hline Hospital length of stay & $18.4 \pm 11.2$ & $15.4 \pm 7.9$ & 0.09 \\
Survivors hospital length of stay & $21.8 \pm 14.6$ & $18.4 \pm 8.1$ & 0.29 \\
Survivors ICU length of stay & $16.8 \pm 16.5$ & $11.8 \pm 9.3$ & 0.22 \\
Survivors duration on ventilator & $10.6 \pm 6.5$ & $7.8 \pm 5.1$ & 0.15 \\
Survivors ventilator to 02 less & $10.5 \pm 6.5$ & $9.0 \pm 6.8$ & 0.52 \\
than 6 L & & & \\
\hline CRRT continu & & & \\
\hline
\end{tabular}

CRRT, continuous renal replacement therapy; CVA, cerebrovascular accident; ICU, intensive care unit.

[HR], 1.18; 95\% confidence interval [CI], 0.71-1.96; $\mathrm{p}=0.516$ ). Underlying renal disease (HR, 2.21; 95\% CI, 1.30-3.75; $\mathrm{p}=0.003$ ) and smoking status (HR, 2.35; 95\% CI; 1.34-4.09; $\mathrm{p}=0.003$ ) were significantly associated with increased mortality. Longer duration of corticosteroid therapy (HR, 0.80; 95\% CI; 0.70-0.90; $<<0.001$ ) and the use of hydroxychloroquine in combination with azithromycin (HR, 0.37; 95\% CI, 0.17-0.81; $\mathrm{p}=0.012$ ) were

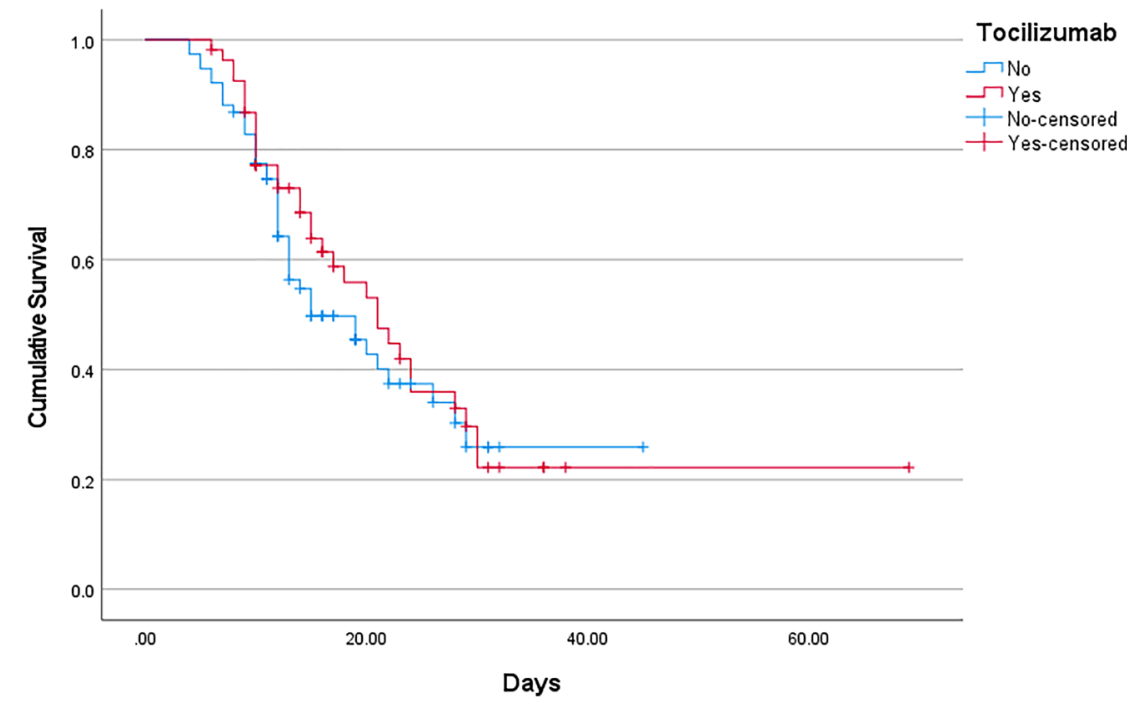

Figure 1: Kaplan-Meier survival curves comparing 30 day survival between Tocilizumab and comparator groups. 
Table 4: Cox regression model for 30 day mortality.

\begin{tabular}{lrr}
\hline Variable & Hazard ratio $(95 \% \mathrm{Cl})$ & $\mathrm{p}$-Value \\
\hline Tocilizumab & $1.18(0.71-1.96)$ & 0.516 \\
Hydroxychloroquine & $0.61(0.33-1.12)$ & 0.109 \\
Hydroxychloroquine + azithromycin & $0.37(0.17-0.81)$ & 0.012 \\
Renal disease $^{\mathrm{a}}$ & $2.21(1.30-3.75)$ & 0.003 \\
Smoking $^{\mathrm{b}}$ & $2.35(1.34-4.09)$ & 0.003 \\
Corticosteroid duration $^{c}$ & $0.80(0.70-0.90)$ & $<0.001$ \\
\hline
\end{tabular}

${ }^{a}$ Renal disease was categorized as having no renal disease or chronic kidney disease stage III-V (including hemodialysis). ${ }^{\text {b Smoking was }}$ categorized as either nonsmoker or smoker (both active and prior history). 'Corticosteroid duration was accounted for as a continuous variable. $\mathrm{Cl}$, confidence interval.

associated with a lower rate of mortality. Multivariate analysis is presented in Table 4 and univariate analysis is presented in the Supplemental Material. Boxplots for corticosteroid duration and 30 day outcomes are presented in the Supplementary Material.

\section{Discussion}

We evaluated the role of TCZ on mortality in critically ill patients with COVID-19. Excess cytokine and chemokine release seen in severe COVID-19 is a dysregulated response of both the innate and adaptive immune system in response to infection caused by SARS-CoV-2. Evidence of hyperinflammation caused by this aberrant host response is seen in association with hypoxic respiratory failure in COVID-19. It has been proposed previously that IL-6 is one of the key drivers of this hyperinflammatory response contributing to the perturbation of the balance, tissue damage, and disease severity [10]. Use of humanized monoclonal antibody against IL- 6 receptor blocks the signaling by this cytokine and reduces inflammation. The use of humanized monoclonal antibody against IL-6 receptor along with corticosteroids is an attempt to decrease inflammation and restore self-regulation of immune system.

Our study showed no survival benefit at 30 days with TCZ vs. comparator treatment in critically ill patients with COVID-19. This is consistent with the results from three prior randomized controlled trials [27-29] that demonstrated no difference in survival rates. In contrast, two other randomized controlled trials $[30,31]$ demonstrated survival benefit; however, in both of those trials, the percentage of patients requiring invasive ventilation at the time of randomization to TCZ treatment was less than $30 \%$, far less than the rate in our cohort of $87.0 \%[30,31]$. Furthermore, in one of those - the Randomized Evaluation of COVID-19 Therapy study [31], which is an ongoing, randomized controlled trial of approximately 4,000 patients - a subsidiary analysis of patients requiring invasive mechanical ventilation at the time of randomization to TCZ treatment revealed no statistically significant difference in mortality or cessation of ventilation [31]. Of the secondary endpoints, we observed significantly lower CRP levels within 48 hours of disposition in the TCZ group (mean CRP, 4.9) than in the comparator group (mean CRP, 13.0; $\mathrm{p}=<0.0001$ ). TCZ blocks IL-6, which has an important role in acute phase response and CRP production [32].

In our cohort, TCZ was administered primarily to patients with critical COVID-19 who required mechanical ventilation. Patients in the TCZ group had higher mean baseline LDH levels, which has been associated with more critical disease and mortality in prior research $[6,33,34]$. Furthermore, the associated in hospital mortality based on APACHE II score at the time of TCZ administration was $25.0 \%$ in survivors compared with $40.0 \%$ in nonsurvivors. Among the 17 patients who received two doses of TCZ, only two survived, and their associated in hospital estimated mortality associated with their APACHE II score was 40.0\% during the first dose and $55.0 \%$ at the time of the second dose. This may reflect the severity or refractoriness of the patients' illness rather than implying a dose dependent toxicity; however, further studies are needed to understand the significance of this finding in our small, critically ill subset of patients.

TCZ has been linked to elevated risk of infection compared with other biological agents, though that study had a much longer duration of treatment than our short term use for CSS in patients with COVID-19 [35]. In our cohort, we did not observe a difference in the overall rate of secondary infection between the two groups. Candidemia was the most common bloodstream infection among patients in the TCZ group; this was also the case in a previous retrospective study of 43 patients [36]. We found that $S$. aureus and $P$. aeruginosa were the most common cause of secondary bacterial pneumonia in patients with COVID-19.

In our cohort, $52 \%$ of patients were smokers, and smoking was associated with increased mortality risk (HR, 2.3; 95\% CI, 1.34-3.74; $\mathrm{p}=0.002$ ). A metanalysis of 11,590 COVID-19 patients demonstrated increased risk of progression of the disease among smokers [37]. In addition, increased risk of ARDS development in smokers has been reported [38, 39]. Elevated IL-6 levels have been shown in both active and former smokers [40]. In our cohort, the mean IL-6 level was higher in smokers than in nonsmokers, though the difference was not statistically significant $(p=0.45)$. The effect of smoking on TCZ efficacy is limited to small studies [41] and larger studies are needed to evaluate it.

Furthermore, our study demonstrated increased mortality among patients with a history of renal disease 
(HR, 2.2; 95\% CI, 1.31-3.75; $\mathrm{p}=0.003$ ). TCZ was shown to be efficacious and safe in a multicenter study of 371 patients with rheumatoid arthritis and concomitant renal insufficiency [42]. In our study, 13 patients in the TCZ group required renal supplemental therapy. This likely reflects the severity of the illness rather than drug toxicity as the onset of AKI occurred mostly prior to TCZ administration.

Our results showed reduced mortality risk in patients who received hydroxychloroquine in combination with azithromycin (HR, 0.37; 95\% CI, 0.17-0.81; $\mathrm{p}=0.012$ ). Hydroxychloroquine inhibits production of IL-1, IL-6, TNF, and IFNy [43], which are also implicated in CSS [11, 12]. Azithromycin, a macrolide antibiotic, has also been shown to inhibit IFN-gamma and TNF-alpha in natural killer cells [44]. Moreover, azithromycin has shown synergistic activity against SARS-CoV-2 when combined with HCQ in vitro [45]. The benefit observed in our study could be related to the immunomodulatory effect of these antimicrobial agents; however, our finding is limited by both the retrospective design and a small sample size $(n=30)$ of patients who received this combined treatment. A randomized, controlled trial of 1,561 patients found no survival benefit at 28 days [46]. Cardiopulmonary arrest was observed in seven patients, and the predominant cause of arrest was worsening hypoxia and shock rather than toxicity related to the specific therapeutics [46].

All patients in our study received corticosteroids for a mean duration of 6.5 days. In our multivariate analysis, longer duration of corticosteroid treatment was associated with reduced mortality risk (HR, 0.80; 95\% CI, 0.71-0.90; $\mathrm{p}=0.0003$ ). Previous authors have suggested that inflammation may recur after abrupt discontinuation of corticosteroid therapy because of a subsequent reconstituted systemic inflammation [47]. There was no statistical difference in the mean corticosteroid duration or timing of administration from admission between the TCZ and comparator groups in our study. Given the recent mortality reduction with corticosteroid use documented in a previous study [19], failure of TCZ to show improved outcomes in our study could have been confounded by the corticosteroid usage. A cohort study [48] of 154 mechanically ventilated patients treated with TCZ found a $45 \%$ reduction in hazard of death; however, overall corticosteroid use in that study was $25 \%$, in contrast to the $100 \%$ rate of use in our cohort.

\section{Limitations}

Our study had several limitations. The first limitation was the method of selection of patients who received TCZ therapy during their hospitalization. This was done utilizing our hospital guidelines, but there was some variability in how patients were selected and hence, our retrospective study population may incorporate some ordering physician bias. Although the data demonstrated very few statistically significant differences between the TCZ and comparator groups, close examination demonstrated higher initial LDH, higher BMI, and trends toward higher IL-6 levels, more frequent invasive ventilation use, more frequent vasopressor use, and longer hospitalization in the patients treated with TCZ, which could represent a bias toward treating the sickest patients with TCZ. This would not be inconsistent nor unexpected for the type of off label use addressed in this study; in addition to having a potential benefit of decreasing inflammation, TCZ use also might come with the adverse burden of additional immunosuppression in patients who are already infected and critically ill.

Additionally, our study was retrospective in nature with a small sample size. About $90 \%$ of the patients who received TCZ in our study had severe disease with respiratory failure requiring mechanical ventilation; therefore, our data did not assess the potential benefit of TCZ in preventing disease progression in patients with less severe disease. Finally, the use of corticosteroid, antimicrobial, and other treatment interventions in our study are confounding factors.

\section{Conclusions}

This cohort study showed no improved survival with the use of TCZ in critically ill patients with COVID-19. Our study did not investigate potential efficacy of TCZ when administered earlier in the clinical course of disease or in a less ill subset of patients with COVID-19. More definitive results from randomized clinical trials are needed to fully assess the potential role of TCZ in the treatment COVID-19.

Research funding: None reported.

Author contributions: Drs. Saffo, Guo, Bhargava, Maksimowicz-McKinnon provided substantial contributions to concesption and design, acquisition of data, or analysis and interpretation of data; Drs. Saffo, MaksimowiczMcKinnon, Guo, Bhargava, J. McKinnon, and Kak drafted the article or revised it critically for important intellectual content; all authors gave final approval of the version of the article to be published; and all authors agree to be accountable for all aspects of the work in ensuring that questions related to the accuracy or integrity of any part of the work are appropriately investigated and resolved.

Competing interests: Dr. Maksimowicz-McKinnon previously served on an advisory board for Genentech. None reported for 
Drs. Saffo, Guo, Bhargava, Kak, J. McKinnon and Ms. Springer have no conflict of interest or financial disclosures related to this work.

Informed consent: A waiver for consent and approval to include vulnerable population data from incarcerated patients was obtained.

Ethical approval: The study was approved by the Henry Ford Institutional Review Board (IRB 13860).

Disclaimer: This study describes off label use of tocilizumab.

\section{References}

1. WHO. Coronavirus disease 2019 (COVID-19) situation report -52 . Available from: www.who.int/docs/default-source/ coronaviruse/situation-reports/20200312-sitrep-52-covid-19. pdf?sfvrsn=e2bfc9c0_4 [Accessed 14 Jun 2020].

2. Dong E, Du H, Gardner L. An interactive web-based dashboard to track COVID-19 in real time. Lancet Infect Dis 2020;20:533-4.

3. Wu Z, McGoogan JM. Characteristics of and important lessons from the coronavirus disease 2019 (COVID-19) outbreak in China: summary of a report of 72314 cases from the Chinese Center for Disease Control and Prevention. JAMA, J Am Med Assoc 2020;312: 1239-442.

4. Richardson S, Hirsch JS, Narasimhan M, Crawford JM, McGinn T, Davidson KW, et al. Presenting characteristics, comorbidities, and outcomes among 5700 patients hospitalized with COVID-19 in the New York City area. JAMA, J Am Med Assoc 2020;323:2052-9.

5. Grasselli G, Greco M, Zanella A, Albano G, Antonelli M, Bellani G, et al. Risk factors associated with mortality among patients with COVID-19 in intensive care units in Lombardy, Italy. JAMA Intern Med 2020;180:1345-55.

6. Wu C, Chen X, Cai Y, Zhou X, Xu S, Huang H, et al. Risk factors associated with acute respiratory distress syndrome and death in patients with coronavirus disease 2019 pneumonia in Wuhan, China. JAMA Intern Med 2020;180:934-43.

7. Mehta P, McAuley DF, Brown M, Sanchez E, Tattersall RS, Manson JJ. COVID-19: consider cytokine storm syndromes and immunosuppression. Lancet 2020;395:1033-4.

8. Xu X, Han M, Li T, Sun W, Wang D, Fu B, et al. Effective treatment of severe COVID-19 patients with tocilizumab. Proc Natl Acad Sci U S A 2020;117:10970-5.

9. Behrens EM, Koretzky GA. Review: cytokine storm syndrome: looking toward the precision medicine era. Arthritis Rheum 2017; 69:1135-43.

10. McGonagle D, Sharif K, O'Regan A, Bridgewood C. The role of cytokines including interleukin- 6 in COVID-19 induced pneumonia and macrophage activation syndrome-like disease. Autoimmun Rev 2020;19:102537.

11. Li Y, Chen M, Cao H, Zhu Y, Zheng J, Zhou H. Extraordinary GU-rich single-strand RNA identified from SARS coronavirus contributes an excessive innate immune response. Microb Infect 2013;15:88-95.

12. Lau SKP, Lau CCY, Chan KH, Li CPY, Chen H, Jin DY, et al. Delayed induction of proinflammatory cytokines and suppression of innate antiviral response by the novel Middle East respiratory syndrome coronavirus: implications for pathogenesis and treatment. J Gen Virol 2013;94:2679-90.

13. de Gans J, van de Beek D. European dexamethasone in adulthood bacterial meningitis study investigators. Dexamethasone in adults with bacterial meningitis. N Engl J Med 2002;347:1549-56.

14. Thwaites GE, Nguyen DB, Nguyen HD, Hoang TQ, Do TTO, Nguyen TCT, et al. Dexamethasone for the treatment of tuberculous meningitis in adolescents and adults. N Engl J Med 2004;351: 1741-51.

15. National Institutes of Health-University of California. Expert Panel for Corticosteroids as Adjunctive Therapy for Pneumocystis Pneumonia. Consensus statement on the use of corticosteroids as adjunctive therapy for pneumocystis pneumonia in the acquired immunodeficiency syndrome. N Engl J Med 1990;323: 1500-4.

16. Meintjes G, Scriven J, Marais S. Management of the immune reconstitution inflammatory syndrome. Curr HIV AIDS Rep 2012; 9:238-50.

17. Stockman LJ, Bellamy R, Garner P. SARS: systematic review of treatment effects. PLoS Med 2006;3:e343.

18. Fadel R, Morrison AR, Vahia A, Smith ZR, Chaudhry Z, Bhargava P, et al. Early short-course corticosteroids in hospitalized patients with COVID-19. Clin Infect Dis 2020;71:2114-20.

19. The RECOVERY Collaborative Group. Dexamethasone in hospitalized patients with covid-19 - preliminary report. N Engl J Med 2020;384:693-704.

20. U.S. Food and Drug Administration. Center for Drug Evaluation and Research. Tocilizumab application number 1252760rig1s114 approval letter. Available from: https://www.accessdata.fda. gov/drugsatfda_docs/nda/2017/1252760rig1s114Approv.pdf [Accessed 14 Jun 2020].

21. Luo P, Liu Y, Qiu L, Liu X, Liu D, Li J. Tocilizumab treatment in COVID-19: a single center experience. J Med Virol 2020;92: 814-8.

22. Toniati P, Piva S, Cattalini M, Garrafa E, Regola F, Castelli F, et al. Tocilizumab for the treatment of severe COVID-19 pneumonia with hyperinflammatory syndrome and acute respiratory failure: a single center study of 100 patients in Brescia, Italy. Autoimmun Rev 2020;19:102568.

23. Colaneri M, Bogliolo L, Valsecchi P, Sacchi P, Zuccaro V, Brandolino F, et al. Tocilizumab for treatment of severe covid-19 patients: preliminary results from smatteo covid19 registry (smacore). Microorganisms 2020;8:695.

24. Capra R, De Rossi N, Mattioli F, Romanelli G, Scarpazza C, Sormani MP, et al. Impact of low dose tocilizumab on mortality rate in patients with COVID-19 related pneumonia. Eur J Intern Med 2020;76:31-5.

25. Sciascia S, Apra F, Baffa A, Baldovino S, Boaro D, Boero R, et al. Pilot prospective open, single-arm multicentre study on off-label use of tocilizumab in patients with severe COVID-19. Clin Exp Rheumatol 2020;38:529-32.

26. Morena V, Milazzo L, Oreni L, Bestetti G, Fossali T, Bassoli C, et al. Off-label use of tocilizumab for the treatment of SARSCoV-2 pneumonia in Milan, Italy. Eur J Intern Med 2020;76: 36-42.

27. Stone JH, Frigault MJ, Serling-Boyd NJ, Fernandes AD, Harvey L, Foulkes A, et al. Efficacy of tocilizumab in patients hospitalized with Covid-19. N Engl J Med 2020;383:2333-44. 
28. Rosas IO, Bräu N, Waters M, Go RC, Hunter BD, Bhagani S, et al. Tocilizumab in hospitalized patients with severe Covid-19 pneumonia. N Engl J Med 2021;384:1503-16.

29. Salama C, Han J, Yau L, Reiss WG, Kramer B, Neidhart JD, et al. Tocilizumab in patients hospitalized with covid-19 pneumonia. N Engl J Med 2021;384:20-30.

30. Gordon AC, Mouncey PR, Al-Beidh F, Rowan KM, Nichol AD, Arabi YM, et al. The RMAP-CAP Investigators. Interleukin- 6 receptor antagonists in critically ill patients with Covid-19. N Engl J Med 2021;384:1491-502.

31. Horby PW, Campbell M, Staplin N, Spata E, Emberson JR, PessoaAmorim G, et al. Recovery Collaborative Group. Tocilizumab in patients admitted to hospital with COVID-19 (RECOVERY): a randomised, controlled, open-label, platform trial. Lancet 2021; 397:1637-45.

32. Schmidt-Arras D, Rose-John S. IL-6 pathway in the liver: from physiopathology to therapy. J Hepatol 2016;64:1403-15.

33. Liang W, Liang H, Ou L, Chen B, Chen A, Li C, et al. Development and validation of a clinical risk score to predict the occurrence of critical illness in hospitalized patients with COVID-19. JAMA Intern Med 2020;180:1081-9.

34. Li C, Ye J, Chen Q, Hu W, Wang L, Fan Y, et al. Elevated Lactate Dehydrogenase (LDH) level as an independent risk factor for the severity and mortality of COVID-19. Aging (NY) 2020;12: 15670-81.

35. Pawar A, Desai RJ, Solomon DH, Ortiz AJ, Gale S, Bao M, et al. Risk of serious infections in tocilizumab versus other biologic drugs in patients with rheumatoid arthritis: a multidatabase cohort study. Ann Rheum Dis 2019;78:456-64.

36. Antinori S, Bonazzetti C, Gubertini G, Capetti A, Pagani C, Morena $V$, et al. Tocilizumab for cytokine storm syndrome in COVID-19 pneumonia: an increased risk for candidemia? Autoimmun Rev 2020;19:102564.

37. Patanavanich R, Glantz SA. Smoking is associated with COVID-19 progression: a meta-analysis. Nicotine Tob Res 2020;22:1653-6.

38. Chaiwat O, Chittawatanarat K, Piriyapathsom A, Pisitsak C, Thawitsri T, Chatmongkolchart $S$, et al. Incidence of and risk factors for acute respiratory distress syndrome in patients admitted to surgical intensive care units: the Multicenter Thai
University-Based Surgical Intensive Care Unit (THAI-SICU) study. J Med Assoc Thail 2016;99:118.

39. Calfee CS, Matthay MA, Kangelaris KN, Siew ED, Janz DR, Bernard $\mathrm{GR}$, et al. Cigarette smoke exposure and the acute respiratory distress syndrome. Crit Care Med 2015;43:1970-7.

40. Aldaham S, Foote JA, Chow HHS, Hakim IA. Smoking status effect on inflammatory markers in a randomized trial of current and former heavy smokers. Int J Inflamm 2015;2015:439396.

41. Theander E, Proven A, Fallang A, Svelander L, Trollmo T. FRI0163 smoking status does not seem to affect tocilizumab efficacy in RA patients. Ann Rheum Dis 2015;74:482.

42. Mori S, Yoshitama T, Hidaka T, Hirakata N, Ueki Y. Effectiveness and safety of tocilizumab therapy for patients with rheumatoid arthritis and renal insufficiency: a real-life registry study in Japan (the ACTRA-RI study). Ann Rheum Dis 2015;74:627-30.

43. Schrezenmeier E, Dörner T. Mechanisms of action of hydroxychloroquine and chloroquine: implications for rheumatology. Nat Rev Rheumatol 2020;16:155-66.

44. Lin SJ, Yan DC, Lee WI, Kuo ML, Hsiao HS, Lee PY. Effect of azithromycin on natural killer cell function. Int Immunopharm 2012;13:8-14.

45. Andreani J, Le Bideau M, Duflot I, Jardot P, Rolland C, Boxberger M, et al. In vitro testing of combined hydroxychloroquine and azithromycin on SARS-CoV-2 shows synergistic effect. Microb Pathog 2020;145:104228.

46. The RECOVERY Collaborative Group. Effect of hydroxychloroquine in hospitalized patients with Covid-19. N Engl J Med 2020;383: 2030-40.

47. Meduri GU, Bridges L, Siemieniuk RAC, Kocak M. An exploratory reanalysis of the randomized trial on efficacy of corticosteroids as rescue therapy for the late phase of acute respiratory distress syndrome. Crit Care Med 2018;46:884-91.

48. Somers EC, Eschenauer GA, Troost JP, Golob JLS, Gandhi TN, Wang $L$, et al. Tocilizumab for treatment of mechanically ventilated patients with COVID-19. Clin Infect Dis 2020:ciaa954. https://doi.org/10.1093/cid/ciaa954.

Supplementary Material: The online version of this article offers supplementary material (https://doi.org/10.1515/jom-2020-0292). 